\title{
Flat supercontinuum generation pumped by amplified noise- like pulses from a figure-eight erbium-doped fiber laser
}

\author{
E Hernández-Escobar ${ }^{1}$, M Bello-Jiménez ${ }^{1}$, O Pottiez ${ }^{2}$, B Ibarra-Escamilla ${ }^{3}$, R López- \\ Estopier $^{1,4}$, M Durán-Sánchez ${ }^{3,4}$ E A Kuzin ${ }^{3}$ and M V Andrés ${ }^{5}$ \\ ${ }^{1}$ Instituto de Investigación en Comunicación Óptica (IICO), Universidad Autónoma de \\ San Luis Potosí, Av. Karakorum No. 1470 Lomas 4ª Secc., 78210 San Luis Potosí, \\ México \\ ${ }^{2}$ Centro de Investigaciones en Óptica (CIO), Loma del Bosque No. 115, Col. Lomas del \\ Campestre, León, Guanajuato 37150, México \\ ${ }^{3}$ Instituto Nacional de Astrofísica, Óptica y Electrónica (INAOE), Luis Enrique Erro No 1, \\ Departamento de Óptica, 72000 Puebla, México \\ ${ }^{4}$ Consejo Nacional de Ciencia y Tecnología (CONACYT), Av. Insurgentes Sur No. 1582, \\ Col. Crédito Constructor, Del. Benito Juárez, México, D.F. 039040, México \\ ${ }^{5}$ Universidad de Valencia, Departamento de Física Aplicada y Electromagnetismo, \\ ICMUV, c/Dr. Moliner 50, Burjassot, 46100 Valencia, Spain \\ E-mail: m.bello@cactus.iico.uaslp.mx
}

\begin{abstract}
The conditions to obtain noise-like pulses (NLPs) from a figure-eight fiber laser (F8L) and their application for supercontinuum (SC) generation in the anomalous dispersion regime are reported. The F8L is designed to remove the undesired low-intensity background radiation from pulse emission, generating NLPs with a 3-dB spectral bandwidth of $17.43 \mathrm{~nm}$ at the fundamental repetition frequency of $0.8 \mathrm{MHz}$. After amplification, NLPs reach a maximum average power of $9.2 \mathrm{~mW}$ and $123.32 \mathrm{~nm}$ spectral bandwidth. By controlling the amplifier pump power, flat SC generation is demonstrated through both a $800-\mathrm{m}$ long spool of SMF-28 fiber and a piece of $5-\mathrm{m}$ long highly nonlinear optical fiber. The results demonstrate a satisfactory flatness of $\sim 3$-dB over a bandwidth of $\sim 1000 \mathrm{~nm}$ in the range from 1261 to $2261 \mathrm{~nm}$, achieving to the best of our knowledge, one of the flattest SC generated from noise-like pulses.
\end{abstract}

Keywords: supercontinuum generation, nonlinear effects.

\section{Introduction}

Fiber lasers capable to produce broad and flat supercontinuum (SC) spectra are very attractive optical sources with important applications in many research fields, such as optical communications, biomedical research, spectroscopy and device characterization, among others [1-7]. Up to now, various fiber-based SC generation schemes have been proposed and demonstrated, relying on different pump light sources [812] and nonlinear optical media [12-18]. Among those approaches, one of the most common method for SC generation relies on injecting ultrashort optical pulses into specialty optical fibers. It was found that the broadest SC spectra are generated when the pump pulse lies in the anomalous dispersion regime, near the zero-dispersion wavelength (ZDW) of the fiber [19]. Under this condition, the broadening mechanism is dominated by soliton dynamics, particularly by the breakup of the injected pulse through soliton fission [20], which is sensitive to input pulse fluctuations and the pump laser shot noise. Consequently, the generated SC is characterized by a complex temporal profile with significant spectral ripple as well as considerable fine structures over its bandwidth [21]. In response to these problems, the use of ideal pedestal-free optical pulses were proposed by Nishizawa et al. to produce high-quality ultra-flat SC spectra. According to their results, the lack of spectral flatness is associated to temporal pedestals of pump pulses in the anomalous dispersion region $[22,23]$. By using ideal pedestal-free Raman shifted solitons in 
a highly nonlinear fiber (HNLF), they reported a high degree of flatness of $\sim 1 \mathrm{~dB}$ for a $520 \mathrm{~nm}$ SC bandwidth in the range from 1370 to $1890 \mathrm{~nm}$ [23]. Since then, many SC schemes with ultra-flat characteristics have been reported based on pedestal-free pump pulses in the normal and anomalous dispersion regimes [24-28].

Recently, mode-locked fiber laser (MLFLs) generating the so-called noise-like pulses (NLPs) have been proposed as the pump sources to realize spectral broadening in different kinds of optical fibers [29-32]. In contrast to standard mode-locked pulses, NLPs are sub-nanosecond wave packets with a fine inner structure of randomly varying pulse durations and peak intensities. This temporal characteristic leads to a double-scaled autocorrelation trace with a sub-picosecond peak riding upon a wide pedestal, suggesting a characteristic of low temporal coherence. Therefore, NLPs find various applications that take advantage of this characteristic, such as optical metrology, optical sensing [33, 34], optical coherence tomography, and optical communications [35-37]. In the spectral domain, NLPs exhibit a smooth and broad output spectrum, which is actually the superposition of incoherent single pulses spectra [38]. By taking advantage of these properties, NLPs have been demonstrated to be an efficient pump source for SC generation [3032]. For example, by using amplified NLPs, Zaytsev et al. demonstrated SC generation through a normally dispersive standard single-mode fiber (SMF), and obtained a SC ranging from 1050 to $1250 \mathrm{~nm}$ [31], and more recently Lin et al. reported SC generation through a HNLF pumped in the anomalous dispersion regime [29], achieving a broad SC spectrum from 1208 to $2111 \mathrm{~nm}$. Nevertheless, in those related investigations, the lack of spectral flatness is a common feature, and spectral variations of up to $20 \mathrm{~dB}$ can be observed in the generated spectra. In this work we focus our attention on the generation of flat SC spectra pumped by amplified NLPs from an erbium-doped figure-eight fiber laser (F8L). The F8L is designed to remove the undesired low-intensity background or pedestal from pulse emission. The results verify that clean NLPs are suitable to generate broad SC with high spectral flatness.

In this paper the conditions to obtain pedestal-free optical pulses from a F8L and their application for SC spectra generation is reported. By simple and clear birefringence adjustments, the laser is capable to operate in various pulse emission regimes, including conventional solitons, NLPs, and simultaneous emission of solitons and NLPs. For the specific case of NLP emission, optical pulses with 3-dB spectral bandwidth of $17.43 \mathrm{~nm}$ were obtained. In order to generate a broad SC spectrum, an erbium/ytterbium $(\mathrm{Er} / \mathrm{Yb})$-doped fiber amplifier is used to boost the power launched into the fiber. By controlling the amplifier pump power, a broad and flat SC generation is demonstrated through both a 800-m long spool of SMF-28 fiber and a short 5-m long piece of highly nonlinear optical fiber (HNLF). The results demonstrate satisfactory uniformity of up to $\sim 3 \mathrm{~dB}$ for a $1000 \mathrm{~nm}$ bandwidth in the range from 1261 to $2261 \mathrm{~nm}$, thus achieving, to our knowledge, one of the flattest SC generated from amplified NLPs.

\section{Experimental setup}

The experimental setup for SC generation is illustrated in figure 1(a). It consists of an Er-based NLP source, a 3-m long Er/Yb double-clad fiber amplifier (CorActive DFC-EY-10/128), and a spool of optical fiber as nonlinear medium. After amplification, broad NLPs with a 3-dB spectral bandwidth of $123.32 \mathrm{~nm}$ and a central wavelength $\left(\lambda_{\mathrm{c}}\right)$ of $1560.8 \mathrm{~nm}$ were obtained. Experiments on SC generation were performed in the anomalous dispersion regime, by launching the amplified NLPs into a spool of 800-m long SMF-28 fiber, with a nonlinear coefficient of $1.5 \mathrm{~W}^{-1} \mathrm{~km}^{-1}$ and ZDW of $1330 \mathrm{~nm}$, and a piece of 5-m long HNLF. The ZDW and nonlinear coefficient of the HNLF are $1548 \mathrm{~nm}$, and $11.3 \mathrm{~W}^{-1} \mathrm{~km}^{-1}$, respectively. The source of NLPs is a mode-locked figure-eight fiber laser (F8L) as illustrated in figure 1(b). It includes a power-symmetrical NOLM located on the right side in the F8L structure. The NOLM consists of a 220-m loop of highly twisted optical fiber, twisted at a rate of 7 turns per meter (twist eliminates the fiber residual birefringence, so that light ellipticity is conserved during propagation), and a quarter-wave retarder (QWR) located asymmetrically into the NOLM loop to provide polarization asymmetry. The QWR is wrapped on a cylinder, we gave special attention to the proper choice for the cylinder diameter and experimentally determined that it has to be $38 \mathrm{~mm}$. Previous researches have shown that this NOLM 
configuration exhibits a flexible transmission that can be modified through adjustments of the QWR angle, or by the NOLM input polarization [39, 40]. Besides, as polarization is controlled both at the NOLM input and inside the loop (through twist and precise QWR adjustment), the switching characteristic can be adjusted precisely and, for given retarder settings, remains fixed over time. In the low-power regime, the NOLM transmission can be adjusted precisely for values between 0 and 0.5 while the transmission at critical power is close to one in any case [39]. The nonlinear transmission of the NOLM is controlled by setting the QWR angle between the maximum and minimum values of the low-intensity transmission. The lowest critical power is achieved at circular input polarization. For this reason a set of polarization elements are used in our setup to introduce at the NOLM input a circular right polarization state. In the low-power regime the NOLM operates as a half-wave plate. For example, assuming circular input polarization, the output polarization is orthogonal to the NOLM input, and the parallel component undergoes zero transmission. However, at high-power levels the parallel component reappears, and undergoes a nonzero nonlinear transmission [41]. To take advantage of the NOLM polarization-dependent transmission, a polarization controller (PC3) followed by a fiber-based polarization beam splitter (PBS) is included at the NOLM output (port P2). The PC3 is adjusted to provide maximum transmission for the low-intensity radiation through one output port of the PBS. This adjustment enables that a small amount of energy can be amplified and fed back to the NOLM input to initiate the mode-locking operation. The remaining output port is used to provide the output light pulses (laser output), with a polarization state associated to high-intensity radiation. This ensures the production of pedestal-free optical pulses without continuous-wave background. A 90/10 coupler is inserted in the cavity to monitor the laser output pulses before the PBS (monitor output). The gain was provided by a 3-m long erbium-doped fiber (EDF). The EDF is pumped through a wavelength division multiplexer coupler (WDM) by a 975-nm pigtailed laser diode, providing a maximum pump power of $101 \mathrm{~mW}$. A set of polarization controllers, PC1 and PC2, are used to control the polarization state at the NOLM input. The PC1 is adjusted to provide maximum transmission through the linear polarizer (LP), and the PC2 is used to introduce a right circular polarization at the NOLM input. A fiber isolator (ISO) is inserted to force unidirectional operation within the laser cavity and to prevent amplification of backward reflections from the NOLM.

(a)

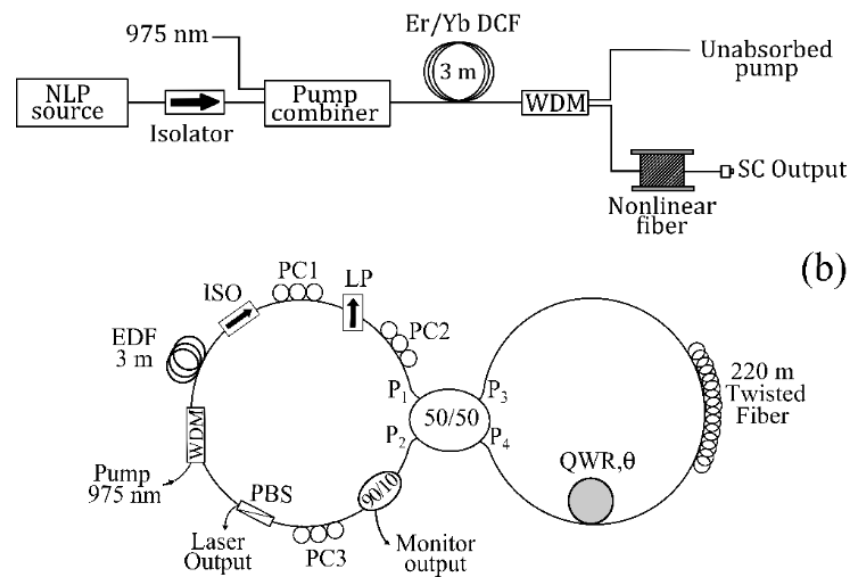

Figure 1. (a) Experimental setup for supercontinuum generation. (b) Schematic setup of the mode-locked figure-eight fiber laser.

Self-starting mode-locking operation was obtained through simple and clear adjustments of the low-power transmission via the QWR angle in the NOLM loop. The required QWR angle was found in the positive slope region of the NOLM transmission curve at low power, as shown in figure 2(a), when the transmission was set to 0.08 . A train of mode-locked pulses appears when pump power is around $90 \mathrm{~mW}$. If pump power is decreased, the number of pulses reduces and the emission of a single pulse is obtained at 
$7.5 \mathrm{~mW}$. This single pulse operation is stable if the pump power is increased until $15 \mathrm{~mW}$. The pulse repetition frequency was measured at $f_{\text {rep }}=0.8 \mathrm{MHz}$, see figure 2(b). Assuming an effective index ( $\left.n_{\text {eff }}\right)$ of 1.4463 the corresponding cavity length, $L_{\text {cavity }}=c /\left(f_{\text {rep }} n_{\text {eff }}\right)$, where $c$ is the speed of light in vacuum, is calculated as $259.28 \mathrm{~m}$. The optical spectra, measured at the monitor (red line) and laser output (black line) are shown in figure 2(c). The full width at half maximum (FWHM) of the spectrum is measured as $1.51 \mathrm{~nm}(\Delta \lambda)$ at the monitor output, centered at $1557.25 \mathrm{~nm}$ with an average output power of $8.33 \mu \mathrm{W}$. As it can be observed, the power spectrum exhibits sharp Kelly sidebands, which confirms that the F8L operates in the soliton regime [42]. With the objective of eliminating low-intensity radiation associated to dispersive waves or pedestals, PC 3 is adjusted to provide maximum transmission for the low-intensity radiation through the PBS port connected to the laser cavity. This ensures the suppression of continuouswave background at the laser output. For proper orientation of PC3, the spectral sidebands disappear as shown in figure 2(c) (black line). From this result, the suppression of intense peaks associated to a continuous-wave background is notable, showing that the pulses are pedestal-free. After suppression of spectral sidebands, a slight increment in spectral bandwidth $(\Delta \lambda)$ is observed, from 1.51 to $1.59 \mathrm{~nm}$, as well as reduction in the average power from $8.33 \mu \mathrm{W}$ to $6.51 \mu \mathrm{W}$. Figure $2(\mathrm{~d})$ shows the corresponding autocorrelation function at the laser output. From these results a pulse duration $\left(T_{\mathrm{FWHM}}\right)$ of 1.53 ps is estimated from the FWHM time delay $\left(T_{\mathrm{ac}}\right)$ of $2.36 \mathrm{ps}\left(T_{\mathrm{FWHM}}=0.648 T_{\mathrm{ac}}\right.$ for solitons). This value along with the spectral bandwidth allows us estimating the time-bandwidth product (TBP) as 0.30. This value is slightly below the transform-limited value of 0.315 for a $\operatorname{Sech}^{2}(\mathrm{x})$ pulse profile; it suggests that pulses from the F8L are close to transform-limited solitons after suppression of spectral sidebands. On the other hand, the emission of NLPs was realized by a small rotation of the QWR angle, while maintaining polarization controllers and pump power fixed. For example, if the QWR angle is rotated to reach a lowpower transmission of 0.1 , as illustrated in figure 2(a), simultaneous emission of NLPs and solitons is generated. Figure 2(e) shows the measured spectrum at both laser outputs, the black line indicates the case where spectral sidebands are suppressed (laser output). Two distinctive pulse emissions can be observed, a broad and smooth optical spectrum centered at $\sim 1549.6 \mathrm{~nm}$, whose characteristics correspond to NLPs emission, and a higher intensity peak centered at $1558.5 \mathrm{~nm}$ corresponding to soliton emission. Moreover, after further rotating the QWR angle, when low-power transmission reaches the value of 0.15 , the spectrum associated to soliton pulses disappears. This result is illustrated in figure 2(f), where a smooth and broad output spectrum is observed, confirming that the F8L is operating in the NLP regime. NLP operation is found for low-power transmission values between 0.15 and 0.21 , for higher values the laser operates in the continuous wave regime. In this scheme, the minimum pump power required to achieve NLP operation was $78 \mathrm{~mW}$. The central wavelength is $1560.8 \mathrm{~nm}$ and the 3 -dB optical bandwidth is 17.43 $\mathrm{nm}$ at the laser output. From these results, the lack of high-energy peaks corresponding to a continuouswave/dispersive wave background is notable, showing that the pulses are pedestal-free. 

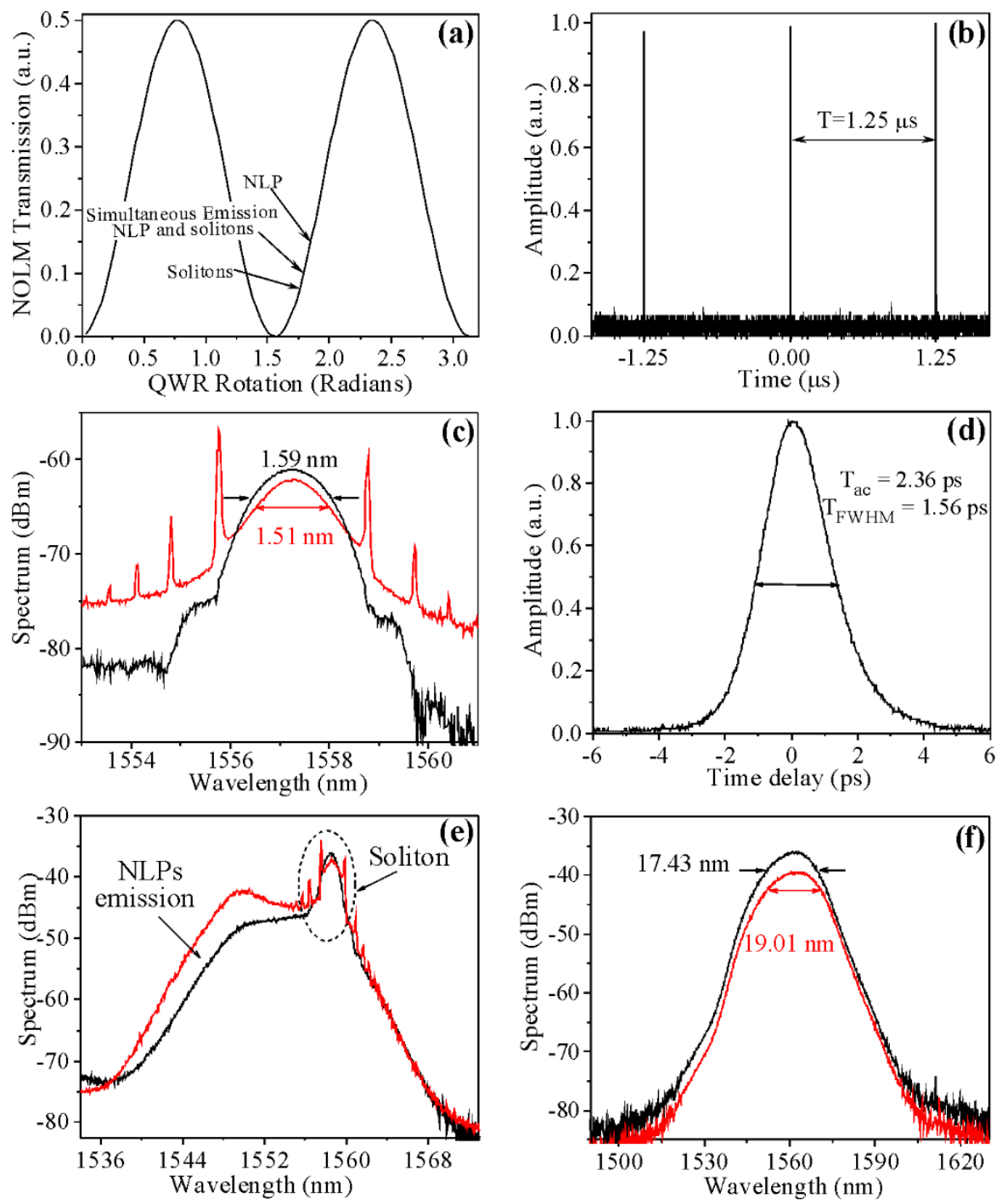

Figure 2. (a) NOLM transmission in low-power regime as a function of the QWR angle. (b) Mode-locked train of pulses generated at $0.8 \mathrm{MHz}$ with $7.5 \mathrm{~mW}$ of pump power. (c) Optical spectrum for single pulse, (d) autocorrelation trace at laser output for single pulse, (e) simultaneous emission of NLPs and solitons. (f) NLP emission. Red and black lines in (c), (e) and (f) indicate emissions at the monitor and laser outputs, respectively.

The corresponding train of NLPs is shown in figure 3(a). A measurement similar to those of conventional single-pulse operation was obtained, with a repetition frequency of $0.8 \mathrm{MHz}$. The average output power is measured as $776.9 \mu \mathrm{W}$ at the maximum pump power of $101 \mathrm{~mW}$. The corresponding autocorrelation function is shown in figure 3(b), measured with a Femtochrome FR-103XL autocorrelator, where a narrow coherent peak and wide shoulders can be observed, with a pedestal-to-peak intensity ratio of about 0.5. This is a typical autocorrelation trace of NLPs including a sub-picosecond peak or coherent spike on top of a broad pedestal. Figure 3(c) shows a close-up view of the coherence spike, confirming that the NLP packet is made of fine temporal sub-structures in the femtosecond (fs) range. If a hyperbolic secant 
profile is assumed, the width of the coherence spike is $~ 204.33$ fs. Figure 3(d) shows the RF spectrum of the output pulses, measured with a $3.2-\mathrm{GHz}$ spectrum analyzer at the fundamental repetition frequency, using a $800 \mathrm{kHz}$ span and $100 \mathrm{~Hz}$ resolution bandwidth. The elevated side bands observed in the spectrum are a clear indication of a considerable amount of amplitude noise encompassed in the trail of the NLPs. As a result, the signal to noise ratio (SNR) of the fundamental frequency peak was measured to be as high as $\sim 45 \mathrm{~dB}$.
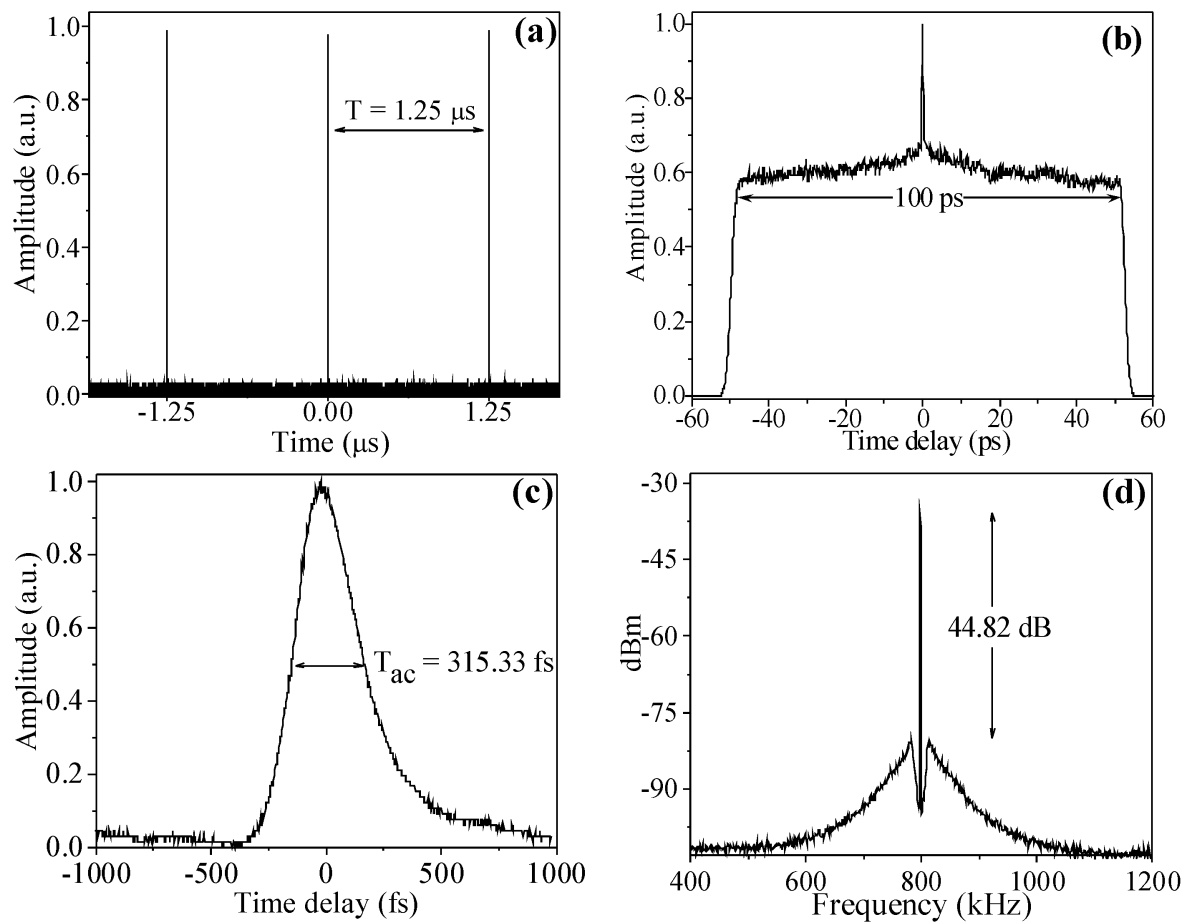

Figure 3. Noise-like pulse operation. (a) Train of pulses generated at $0.8 \mathrm{MHz}$ with 101-mW pump power. (b) Autocorrelation trace (100 ps corresponds to the measurement window). (c) Zoom-in of the coherence spike of the autocorrelation trace. (d) RF spectrum of the output pulse train measured with $800 \mathrm{kHz}$ span and $100 \mathrm{~Hz}$ resolution bandwidth.

The characteristics of amplified NLPs are illustrated in figure 4. The evolution of NLP spectrum as a function of amplifier pump power is shown in figure 4(a), where a spectrum enhancement of up to 123.32 $\mathrm{nm}$ can be observed at the maximum amplifier pump power of $2.74 \mathrm{~W}$. At this value of amplification the spectrum broadens significantly, reaching an improvement factor of $\times 7$ at 3 -dB bandwidth. It indicates that the NLP experiences significant nonlinear optical effects during the amplification process. The spectrum shape reveals the compression of NLPs at the initial stage of amplification followed by a red shift in wavelength produced by intrapulse Raman scattering (IRS). The measured average power after amplification in conjunction with the 3-dB spectral bandwidth is shown in figure 4(b). A maximum average power of $9.2 \mathrm{~mW}$ can be achieved at the maximum amplifier pump power of $2.74 \mathrm{~W}$, which corresponds to a maximum amplification of $10.75 \mathrm{~dB}$. Finally, temporal characteristics of amplified NLPs are shown in figures $4(\mathrm{c})$ and $4(\mathrm{~d})$ at the amplifier pump power of $2.74 \mathrm{~W}$. A similar double-scaled autocorrelation trace is observed with a pedestal-to-peak intensity ratio of 0.7 , suggesting a similar noiselike characteristic after amplification. However, the peak duration of autocorrelation trace reduces significantly down to $173.5 \mathrm{fs}$, suggesting that the temporal durations of the inner pulses of amplified noise-like pulses are similarly shortened, reaching a compression ratio of about 0.85 . 
(a)
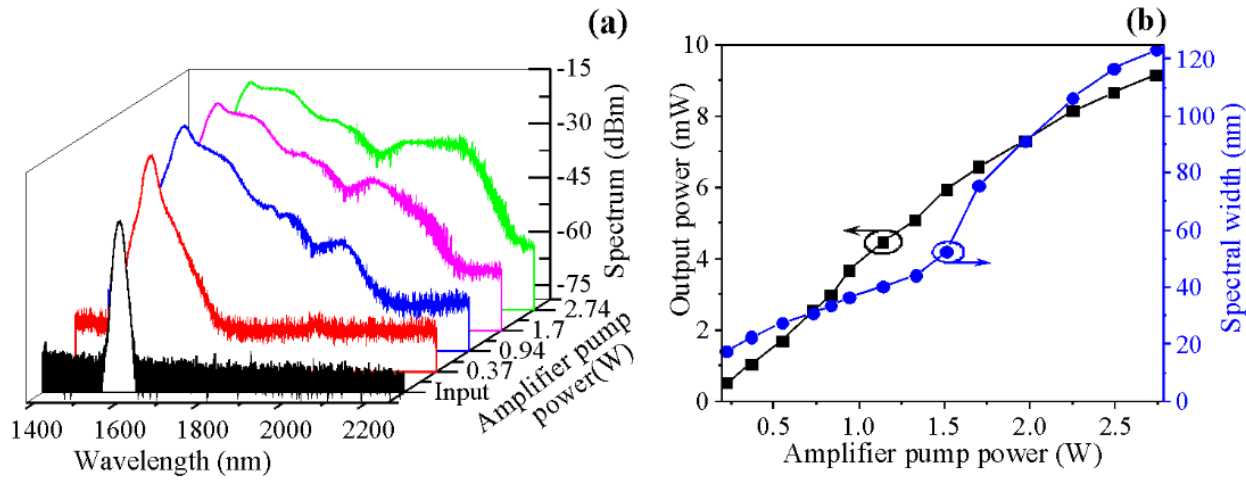

(c)
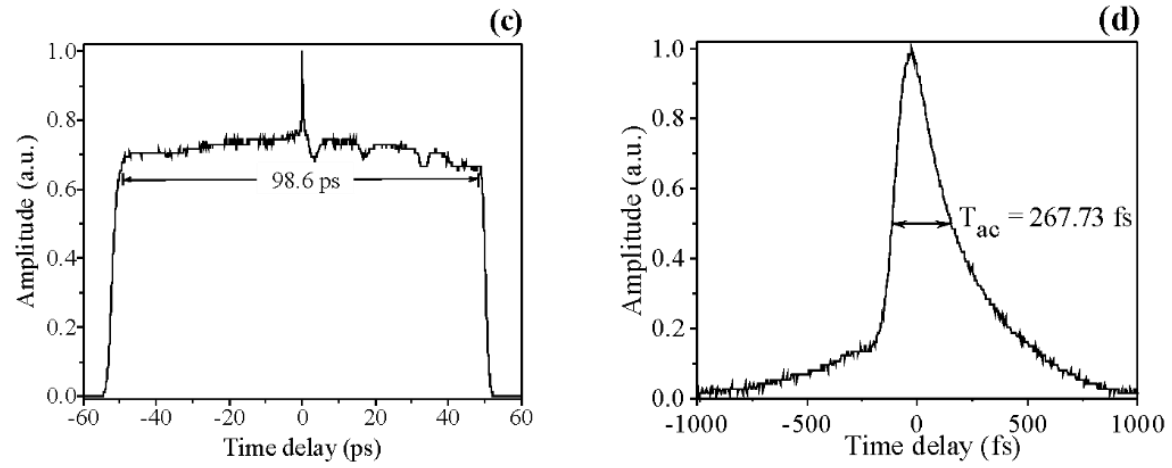

Figure 4. (a) Spectrum evolution of NLPs as the amplifier pump power increases. (b) Output power and 3-dB bandwidth of amplified NLPs. (c) Autocorrelation trace at the maximum amplifier pump power of $2.74 \mathrm{~W}$ and (d) Zoom-in of the coherence peak of the autocorrelation trace.

As can be seen from above results, the capacity to compress NLPs through the amplification process provides the advantage of generating a significantly broader emission. For our particular results, the amplification of NLPs demonstrate a spectral improvement from 17.43 to $123.32 \mathrm{~nm}$, covering a full spectral range from 1390 to $2172 \mathrm{~nm}$. This property makes NLPs very attractive for applications such as SC generation in the mid-infrared pumped by erbium fiber lasers. In the following section, SC generation by amplified NLPs is demonstrated in standard and highly nonlinear optical fibers.

\section{Experimental results and discussion}

Experiments on SC generation were performed in the anomalous dispersion regime by pumping two different types of optical fibers. The first set of experiments was realized using a spool of $800-\mathrm{m}$ long single-mode optical fiber (SMF-28). In order to control the average power of amplified NLPs, the amplifier pump power is varied to reach a maximum average power of $9.2 \mathrm{~mW}$. Figure 5(a) shows the evolution of the generated SC spectra at different amplifier pump powers. As it can be observed, the higher average input power (also corresponding to the broader input spectrum) produces the wider and flatter SC spectrum. This result is in agreement with observations reported in the literature using amplified NLPs [31, 32]. Note that spectral broadening due to Raman self-frequency shift (SFS) is reasonably uniform, in particular for the spectral range between 1534.10 and $1811 \mathrm{~nm}$, where a spectral flatness of 4 $\mathrm{dB}$ is observed. Beyond this spectral range, a resonant peak appears centered at $2010.1 \mathrm{~nm}$, which looks like one of the special four-wave mixing processes favored by some particular phase matching condition recently reported [43]. To quantify the spectral bandwidth, the 10 and 20-dB spectral widths are measured for comparison and analysis. Figure 5(b) presents the spectral width as a function of amplifier pump power. At the launched power of $9.2 \mathrm{~mW}$, a SC spectrum with $537.25 \mathrm{~nm}$ bandwidth ranging from 1508 
to $2045.25 \mathrm{~nm}$ is generated. Figure 5(c) gives a detailed picture of the generated SC at the maximum power launched into the fiber.

(a)

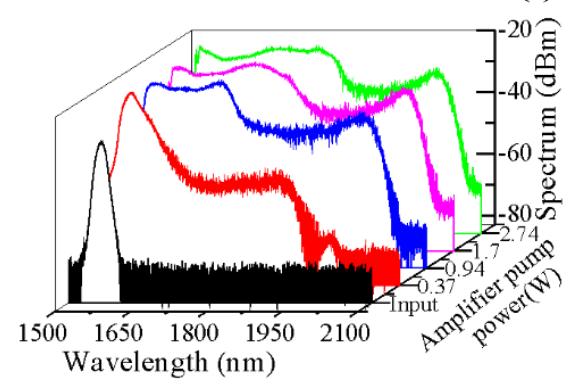

(b)

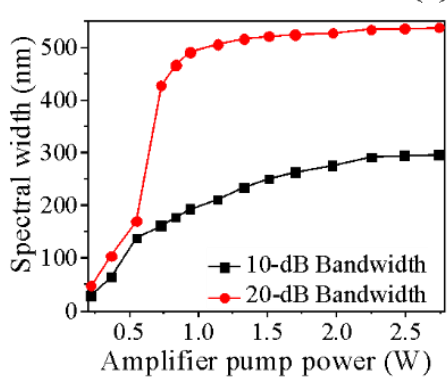

(c)

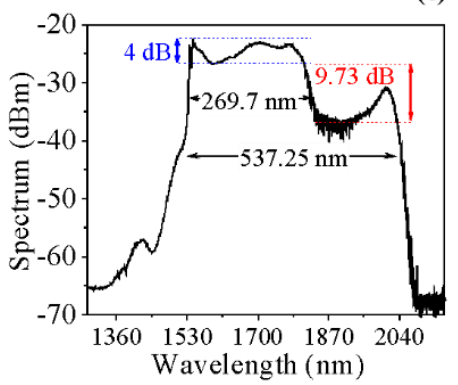

Figure 5. Supercontinuum generation in a spool of 800-m long SMF-28 fiber. (a) SC evolution as a function of amplifier pump power. (b) Measured 10- and 20-dB spectral width of generated SC spectrum. (c) SC spectrum generated at $2.74 \mathrm{~W}$ of amplifier pump power.

After analysis of the SMF-28 fiber, the amplifier was spliced to a 5-m long HNLF with ZDW at $1548 \mathrm{~nm}$ and nonlinear parameter of $11.3 \mathrm{~W}^{-1} \mathrm{~m}^{-1}$. Figure 6(a) shows the generated spectrum as a function of the amplifier pump power. As the figure indicates, the SC spectrum broadened as the pump power increased, exhibiting a spectral shape that remains smooth at higher power levels. With the maximum launched power of $9.2 \mathrm{~mW}$, which corresponds to an amplifier pump power of $2.74 \mathrm{~W}$, a SC with average power of $2.45 \mathrm{~mW}$ was obtained. The SC ranged from 1261 to $2261 \mathrm{~nm}$ in the 20-dB bandwidth, with a spectral flatness of $\sim 3 \mathrm{~dB}$ over almost the entire spectrum. The fine structure around $1900 \mathrm{~nm}$ is demonstrated to be caused by the molecular resonances of $\mathrm{H}_{2} \mathrm{O}$ and $\mathrm{CO}_{2}$ [44]. Figure 6(b) presents the spectral width measured at 10 and $20-\mathrm{dB}$ bandwidth as a function of amplifier pump power. Starting well below the maximal launched power of $9.2 \mathrm{~mW}$, both bandwidths saturate and become similar due to the high degree of spectral flatness in the output spectrum. Figure 6(c) gives a detailed picture of the flattest supercontinuum generated at $1.7 \mathrm{~W}$ of amplifier pump power.

(a)

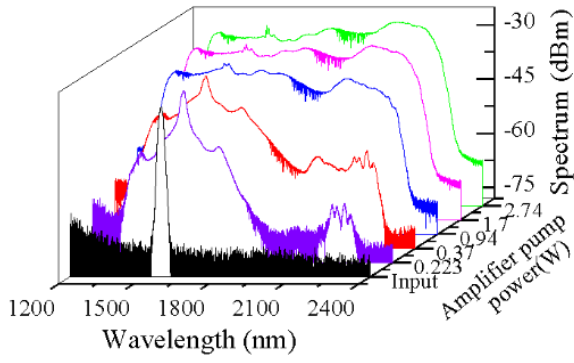

(b)

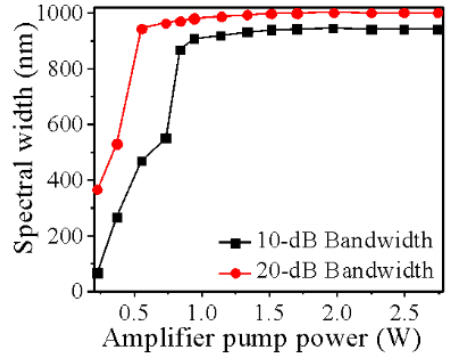

(c)

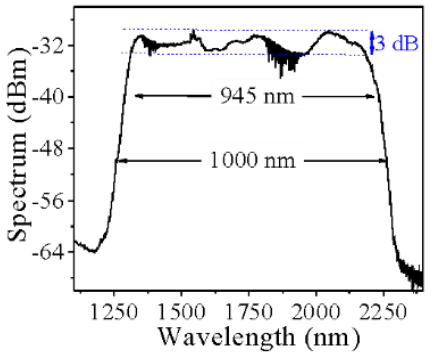

Figure 6. Supercontinuum generation in a 5-m long HNLF. SC evolution as a function of amplifier pump power. (b) Measured 10 and 20-dB spectral width of generated SC spectrum. (c) Flattest supercontinuum generated at $1.7 \mathrm{~W}$ of amplifier pump power.

For comparison purposes, and to show the improvement achieved with the present scheme, SC spectrum is measured under the same conditions reported in figure 6(c), but in this case the polarization controller PC3 is adjusted to allow the transmission of low-intensity radiation at the laser output, see figure 1(b). The characteristics of the resulting NLPs are depicted in figures 7(a) and 7(b). The central wavelength is $1560 \mathrm{~nm}$ and the $-3 \mathrm{~dB}$ optical bandwidth is $9.73 \mathrm{~nm}$. In comparison to the spectrum obtained in figure 2(f) (black line), these NLPs exhibit a peak of amplified spontaneous emission at 1530 $\mathrm{nm}$, suggesting the presence of low-intensity radiation. In addition, the pedestal-to-peak intensity ratio reaches a higher value of 0.65 . At the amplifier pump power of $1.7 \mathrm{~W}$, a SC spectrum with $\sim 1000 \mathrm{~nm}$ 
bandwidth ranging from 1250 to $2250 \mathrm{~nm}$ is generated. The detailed picture of the SC spectrum is illustrated in figure 7(c), where spectral oscillations of up to $\sim 30 \mathrm{~dB}$ can be observed. If we compare this result with the SC spectrum obtained in figure 6(c), where clean NLPs were used, we can conclude that pedestal-free NLPs are best suited for broad SC generation with high degree of flatness.
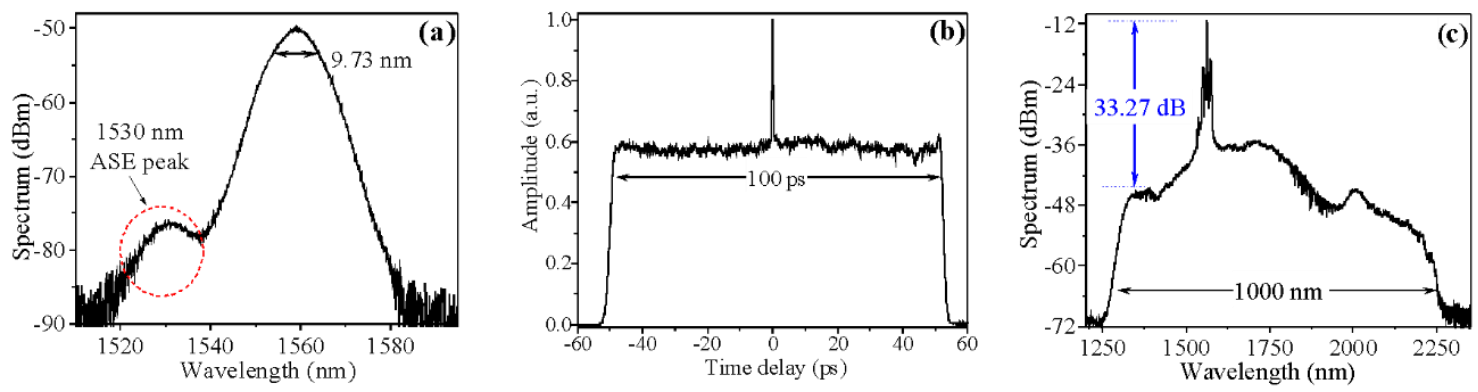

Figure 7. Characteristics of NLPs with low-intensity radiation and SC generation. (a) Optical spectrum of NLPs. (b) Autocorrelation trace (100 ps corresponds to the measurement window). (c) $\mathrm{SC}$ spectrum generated at $1.7 \mathrm{~W}$ of amplifier pump power.

From these results, the generation of broad and flat SC spectra pumped by amplified NLPs is demonstrated in the anomalous dispersion regime. With a 5-m long HNFL, we demonstrate an excellent flatness of $\sim 3 \mathrm{~dB}$ for the $1000 \mathrm{~nm}$ bandwidth in the spectral range from 1261 to $2261 \mathrm{~nm}$, thus achieving, to our knowledge, one of the flattest SC sources pumped by NLPs. We believe that SC schemes based on NLPs are very attractive optical sources for applications requiring low temporal coherence.

\section{Conclusions}

The conditions to obtain pedestal-free optical pulses from a F8L and its application for SC generation are reported. The proposed F8L takes advantage of the NOLM polarization-dependent transmission to eliminate the low-intensity background at the laser output. By simple and clear adjustments of the QWR angle into the NOLM loop, the F8L is capable to operate in various pulse emission regimes, including conventional solitons, NLPs, and simultaneous emission solitons and NLPs. For the specific case of NLP emission, optical pulses with $3-\mathrm{dB}$ spectral bandwidth of $17.43 \mathrm{~nm}$ were obtained at the fundamental repetition frequency of $0.8 \mathrm{MHz}$. In order to generate a broad SC spectrum, an Er/Yb-doped fiber amplifier is used to boost the power launched into the fiber. After amplification, NLPs reach a maximum average power of $9.2 \mathrm{~mW}$ and a $123.32 \mathrm{~nm}$ spectral bandwidth, corresponding to a pulse energy of 11.5 nJ. By controlling the amplifier pump power, our best result demonstrates a broad and flat SC generation through a 5-m long HNLF. The source exhibits an excellent flatness of $\sim 3 \mathrm{~dB}$ for a $1000 \mathrm{~nm}$ bandwidth in the range from 1261 to $2261 \mathrm{~nm}$. The results verify that clean NLPs are suitable to generate broad SC spectra with a high degree of flatness.

\section{Acknowledgments}

This work was supported by CONACyT Grant 222476 and "Fronteras de la ciencia" grant 2016-01-2438.

\section{References}

[1] Kawanishi S, Takara H, Uchiyama K, Shake I and Mori K 19993 Tbit/s (160 Gbit/s 19 channel) optical TDM and WDM transmission experiment Electron. Lett. 35 826-7

[2] Takara H, Ohara T, Mori K, Sato K, Yamada E, Inoue Y, Shibata T, Abe M, Morioka T and Sato K I 2000 More than 1000 channel optical frequency chain generation from single supercontinuum source with $12.5 \mathrm{GHz}$ channel spacing Electron. Lett. 36 2089-90 
[3] Courvoisier C, Mussot A, Bendoula R, Sylvestre T, Reyes J G, Tribillon G, Wacogne B, Gharbi T and Maillotte H 2004 Broadband supercontinuum in a microchip-laser-pumped conventional fiber: Toward biomedical applications," Laser Phys. 14 507-14

[4] Kaminski C F, Watt R S, Elder A D, Frank J H and Hult J 2008 Supercontinuum radiation for applications in chemical sensing and microscopy Appl. Phys. B 92 367-78

[5] Ruehl A et al J 2011 Ultrabroadband coherent supercontinuum frequency comb Phys. Rev. A 84 11806-11

[6] Udem Th, Holzwarth R and Hansch T W 2002 Optical frequency metrology Nature 416 233-7

[7] Isobe K, Watanabe W, Matunaga S, Higashi T, Fukui K and Itoh K 2005 Multi-spectral two-photon excited fluorescence microscopy using supercontinuum light source J. Appl. Phys. 44 L167-9

[8] Swiderski J and Michalska M 2013 The generation of a broadband, spectrally flat supercontinuum extended to the midinfrared with the use of conventional single-mode fibers and thulium-doped single-mode fibers pumped by $1.55 \mu \mathrm{m}$ pulses Laser Phys. Lett. 10015106

[9] Geng J, Wang Q and Jiang S 2012 High-spectral-flatness mid-infrared supercontinuum generated from a Tm-doped fiber amplifier Appl. Optics $\mathbf{5 1} 834-40$

[10] Swiderski J, Theberge F, Michalska M, Mathieu P and Vincent D 2014 High average power supercontinuum generation in a fluoroindate fiber Laser Phys. Lett. 11015106

[11] Song R, Hou J, Chen S P, Yang W Q, Liu T and Lu Q S 2013 Near-infrared supercontinuum generation in an all-normal dispersion MOPA configuration above one hundred watts Laser Phys. Lett. 10015401

[12] Hernandez-Garcia J C, Estudillo-Ayala J M, Mata-Chavez R I, Pottiez O, Rojas-Laguna R and Alvarado-Mendez E 2013 Experimental study on a broad and flat supercontinuum spectrum generated through a system of two PCFs Laser Phys. Lett. 10075101

[13] Zhu H, Wang R, Pu T, Fang T, Xiang P, Zheng J and Chen D 2015 Optical stealth transmission based on supercontinuum generation in highly nonlinear fiber over WDM network Opt. Lett. 40 2561-3

[14] Quang N, Matsuura M and Kishi N 2014 WDM-to-OTDM conversion using supercontinuum generation in a highly nonlinear fiber IEEE Photonic. Tech. L. 26 1882-5

[15] Tripathy S K, Achary J S N, Muduli N and Palai G 2015 Nonlinear rectangular photonic crystal fiber (PCF) for optical communication exclusively super continuum generation J. Laser Opt. Photonics 21000114

[16] Jiang X, Joly N Y, Finger M A, Babic F, Wong G K, Travers J C and Russell P S J 2015 Deep-ultraviolet to midinfrared supercontinuum generated in solid-core ZBLAN photonic crystal fibre Nat. Photonics 9 133-9

[17] Anashkina E A, Andrianov AV, Yu. Koptev M, Muravyev S V and Kim A V 214 Generating femtosecond optical pulses tunable from 2 to $3 \mu \mathrm{m}$ with a silica-based all-fiber laser system Opt. Lett. 39 2963-6

[18] Kamynin V A, Bednyakova A E, Fedoruk M P, Volkov I A, Nishchev K N and Kurkov A S 2015 Supercontinuum generation beyond $2 \mu \mathrm{m}$ in $\mathrm{GeO}_{2}$ fiber: comparison of nano- and femtosecond pumping Laser Phys. Lett. 12065101

[19] Dudley J M, Genty G and Coen S 2006 Supercontinuum generation in photonic crystal fiber Rev. Mod. Phys. 78 113584

[20] Herrmann J, Griebner U, Zhavoronkov N, Husakou A, Nickel D, Knight J C, Wadsworth W J, Russell P St J and Korn G 2002 Experimental evidence for supercontinuum generation by fission of higher-order solitons in photonic fibers Phys. Rev. Lett. 88173901

[21] Gu X, Xu L, Kimmel M, Zeek E, O’Shea P, Shreenath A P, Trebino R and Windeler R S 2002 Frequency resolved optical gating and single-shot spectral measurements reveal fine structure in microstructure-fiber continuum Opt. Lett. 27 1174-6

[22] Nishizawa N and Hori M 2008 Octave spanning high quality super continuum generation using $10 \mathrm{~nJ}$ and $104 \mathrm{fs}$ high energy ultrashort soliton pulse Appl. Phys. Express 1022009

[23] Nishizawa N and Takayanagi J 2007 Octave spanning high-quality supercontinuum generation in all-fiber system $J$. Opt. Soc. Am. B 24 1786-92

[24] Qin G, Yan X, Kito C, Liao M, Chaudhari C, Suzuki T and Ohishi Y 2009 Ultrabroadband supercontinuum generation from ultraviolet to $6.28 \mu \mathrm{m}$ in a fluoride fiber Appl. Phys. Lett. 95161103

[25] Miret J J, Silvestre E and Andrés P 2009 Octave-spanning ultraflat supercontinuum with soft-glass photonic crystal fibers Opt. Express 17 9197-203

[26] Heidt A M 2010 Pulse preserving flat-top supercontinuum generation in all-normal dispersion photonic crystal fibers 2010 J. Opt. Soc. Am. B 27 550-9

[27] Nozaki Y, Nomura Y, Aramaki M and Nishizawa N 2014 Octave spanning coherent supercontinuum generation using 51 fs high-power ultrashort pulse from Er-doped similariton amplifier Japanese Journal of Applied Physics $\mathbf{5 3} 020301$

[28] Liao R, Song Y, Zhou X, Chai L, Wang Cand Hu M 2016 Ultra-flat supercontinuum generated from high-power, picosecond telecommunication fiber laser source Appl. Opt. $\mathbf{5 5} 9384-8$ 
[29] Lin S S, Hwang S K and Liu J M 2014 Supercontinuum generation in highly nonlinear fibers using amplified noise-like optical pulses Opt. Express 22 4152-60

[30] Kobtsev S, Kukarin S, Smirnov S and Ankudinov I 2014 Cascaded SRS of single- and double-scale fiber laser pulses in long extra-cavity fiber Opt. Express 22 20770-5

[31] Zaytsev A, Lin C H, You Y J, Chung C C, Wang C L and Pan C L 2013 Supercontinuum generation by noise-like pulses transmitted through normally dispersive standard single-mode fibers Opt. Express 21 16056-62

[32] Chen H, Zhou X, Chen S P, Jiang Z F and Hou J 2015 Ultra-compact Watt-level flat supercontinuum source pumped by noise-like pulse from an all-fiber oscillator Opt. Express 23 32909-16

[33] Goloborodko V, Keren S, Rosenthal A, Levit B and Horowitz M 2003 Measuring temperature profiles in high power optical fiber components Appl. Opt. 42 2284-8

[34] Putnam M A, Dennis M L, Duling I N, Askins C G and Friebele E J 1998 Broadband square-pulses operation of passively mode-locked fiber laser for fiber gratings interrogation Opt. Lett. 23 138-40

[35] Keren S, Brand E, Levi Y, Levit B and Horowitz M 2002 Data storage in optical fibers and reconstruction by use of low-coherence spectral interferometry Opt. Lett. 27 125-7

[36] Keren S and Horowitz M 2001 Interrogation of fiber gratings by use of low-coherence spectral interferometry of noiselike pulses Opt. Lett. 26 328-33

[37] Keren S, Rosenthal A and Horowitz M 2003 Measuring the structure of highly reflecting fiber Bragg grating IEEE Photonics Technol. Lett. 15 575-7

[38] Runge A F J, Aguergaray C, Broderick N G R and Erkintalo M 2013 Coherence and shot-to-shot spectral fluctuations in noise-like ultrafast fiber lasers Opt. Lett. 38 4327-30

[39] Pottiez O, Kuzin E A, Ibarra-Escamilla B and Méndez-Martínez F 2005 Theoretical investigation of the NOLM with highly twisted fibre and a $\lambda / 4$ birefringence bias Opt. Commun. 254 152-67

[40] Ibarra-Escamilla B, Kuzin E A, Zaca-Moran P, Grajales-Coutiño R, Mendez-Martinez F, Pottiez O, Rojas-Laguna R and Haus J W 2005 Experimental investigation of the nonlinear optical loop mirror with twisted fiber and birefringence bias Opt. Express 13 10760-7

[41] Ibarra-Escamilla B, Pottiez O, Haus J W, Kuzin E A, Bello-Jimenez M and Flores-Rosas A 2008 Wavelength-tunable picosecond pulses from a passively mode-locked figure-eight Erbium-doped fiber laser with a Sagnac fiber filter $J$. Eur. Opt. Soc. $\mathbf{3} 08036$

[42] Kelly S M J 1992 Characteristic sideband instability of periodically amplified average soliton Electron. Lett. 28 806-7

[43] Castelló Lurbe D, Vermeulen N and Silvestre E 2016 Towards an analytical framework for tailoring supercontinuum generation Opt. Express 24 26629-45

[44] Rothman L S et al 2013 The HITRAN 2012 molecular spectroscopic database J. Quant. Spectrosc. Radiat. Transf. 130, $4-50$ 